\title{
Tuning of Tungsten Thin Film Superconducting Transition Temperature for Fabrication of Photon Number Resolving Detectors
}

\author{
A. E. Lita, D. Rosenberg, S. Nam, A. J. Miller, D. Balzar, L. M. Kaatz, and R. E. Schwall
}

\begin{abstract}
Tungsten thin films can form in one of two crystal structures: alpha (bcc), with a superconducting transition temperature $\left(T_{\mathrm{c}}\right)$ of $15 \mathrm{mK}$, and beta (A15), with a $T_{\mathrm{c}}$ between 1 and $4 \mathrm{~K}$. Films with intermediate $T_{\mathrm{c}} \mathrm{s}$ are composed of both alpha and beta phases. We have investigated how to tune the film $T_{\mathrm{c}}$ in order to obtain certain values $\left(T_{\mathrm{c}} \sim 100 \mathrm{mK}\right)$ suitable for the fabrication of photon number resolving transition-edge sensor (TES) and arrays of TES detectors for astronomical and quantum information applications. Variation of deposition conditions, and also the choice of the underlayer/coating for equal deposition conditions, affect the $T_{\mathrm{c}} \mathrm{s}$ of tungsten films. We have used $\mathrm{x}$-ray diffraction to determine the structure of tungsten thin films and film stress. The results indicates that the film stress state depends on the underlying substrate and coating. To understand the variation of $T_{\mathrm{c}}$ values and to allow precise tuning of these values, we have investigated substrates and coatings for tungsten film multilayer stacks and determined tungsten film stress by $x$-ray diffraction at both room temperature and $8 \mathrm{~K}$.
\end{abstract}

Index Terms-Materials processing, stress, superconducting films, x-ray measurements.

\section{INTRODUCTION}

$\mathbf{T}$ UNGSTEN is the material of choice for transition edge sensors (TESs) for optical and near-infrared wavelengths due to the tunability of its superconducting transition temperature $\left(T_{\mathrm{c}}\right)$ in the $\sim 100 \mathrm{mK}$ range and the relative weak coupling between its electron and phonon systems at these temperatures [1], [2]. By exploiting the superconducting properties of tungsten (W) thin films, these devices become extremely sensitive thermometers, making TESs arguably the most sensitive photon number resolving detectors in existence [3]. The $T_{\mathrm{c}}$ values of thin $\mathrm{W}$ films vary based on the film microstructure. As early as 1965 , it has been shown that $\mathrm{W}$ films containing $\beta$-W structure display transition temperatures two orders of magnitude higher compared to reported values for bulk $\alpha-\mathrm{W}$ [4], [5]. Sputtered $\mathrm{W}$ thin films typically consist of single-phase $\alpha$-W (bcc) with $T_{\mathrm{c}}=15 \mathrm{mK}$, single-phase $\beta$-W (A15) with a $T_{\mathrm{c}}$ between 1 and $4 \mathrm{~K}$, or a mixture of these two phases with a $T_{\mathrm{c}}$ dependent on

Manuscript received October 5, 2004. This work was supported in part by ARDA under Contract MOD 7084.04.

A. E. Lita, D. Rosenberg, S. Nam, A. J. Miller and R. E. Schwall are with the National Institute of Standards and Technology (NIST), Boulder, CO USA (e-mail: lita@ boulder.nist.gov).

D. Balzar is with the National Institute of Standards and Technology (NIST), Boulder, CO USA and also with the Department of Physics and Astronomy, University of Denver, Denver, CO USA.

L. M. Kaatz is with the Department of Physics and Astronomy, University of Denver, Denver, CO USA

Digital Object Identifier 10.1109/TASC.2005.849033 the ratio of the two phases, as indicated in this paper. The film structure has been reported to depend strongly on the deposition conditions [5]-[8]. Because $\mathrm{W}$ is widely used in the semiconductor industry for interconnects, diffusion barriers, and seed layers, correlations between residual stress, microstructure, and surface morphology have been widely investigated. However, up to now these properties have not been related to $T_{\mathrm{c}}$.

We were able to reliably predict the film $T_{\mathrm{c}}$ as a function of the deposition conditions for our sputtering system, through a design of experiment approach. We have used x-ray diffraction (XRD) measurements to correlate $\mathrm{W}$ film $T_{\mathrm{c}}$ values with both phase composition and film stress. The choice of the underlayer and coating of the $\mathrm{W}$ thin film also appeared to have a strong effect on the $\mathrm{W}$ film $T_{\mathrm{c}}$.

However, the room-temperature x-ray diffraction studies revealed no significant changes in phase composition even when the $T_{\mathrm{c}}$ values changed by $100 \mathrm{mK}$. Also, the stress values measured at room temperature did not explain the $T_{\mathrm{c}}$ variation. This indicates that the changes in $T_{\mathrm{c}}$ may be caused by stress induced through differential thermal contraction of the underlayer/coating and/or changes in the phase composition at cryogenic temperatures relative to room temperatures. We have performed $\mathrm{x}$-ray diffraction measurements at $8 \mathrm{~K}$ that have shown that the underlayer and/or coating strongly affect the film stress through differential thermal contraction. A correlation has been proposed between the $\mathrm{W}$ film $T_{\mathrm{c}}$ and the film stress as a result of differential thermal contraction.

\section{EXPERIMENTAL PROCEDURE}

Thin $(20 \mathrm{~nm})$ films of $\mathrm{W}$ were deposited by dc magnetron sputtering from a 3 inch diameter W cathode target $(99.999 \%$ purity) located $12 \mathrm{~cm}$ from the sample. The chamber base pressure was typically $1 \times 10^{-5} \mathrm{~Pa}$. The substrates used were 3 inch diameter (100) Si wafers ( $350 \mu \mathrm{m}$ thick) with native oxide. The W film thicknesses were determined by measuring a patterned step height using a stylus-type surface profiler. The $20 \mathrm{~nm}$ thickness provides the highest TES efficiency when $\mathrm{W}$ films are incorporated in an optical cavity [2].

Following deposition, the sheet resistance was measured with a four-point probe, and the film stress was measured based on changes in the substrate curvature.

The $T_{\mathrm{C}} \mathrm{S}$ were measured by use of an adiabatic demagnetization refrigerator with a base temperature of $60 \mathrm{mK}$. The samples were voltage biased and the output current was read out with a SQUID array amplifier [9], [10]. 


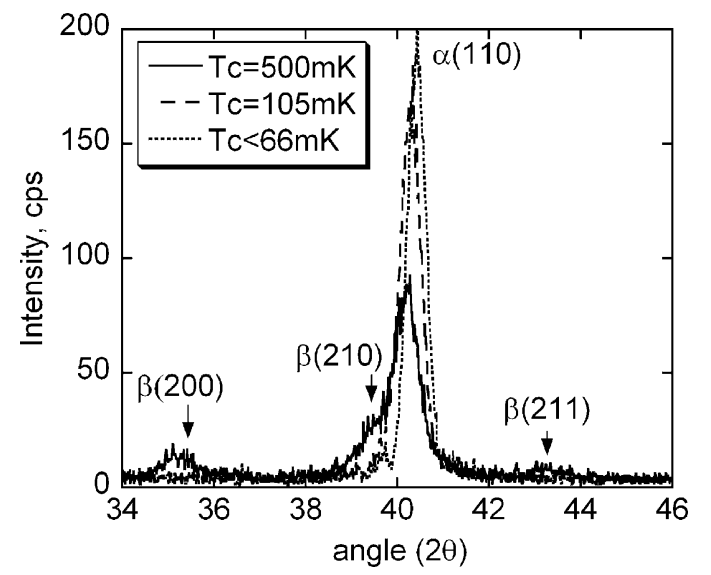

Fig. 1. Correlation of $\mathrm{W}$ films $T_{\mathrm{c}}$ to phase composition.

The XRD measurements were carried out with a diffractometer with a $\mathrm{Cu}$ tube operated at $45 \mathrm{kV}$ and $40 \mathrm{~mA}$. A Ge solid-state detector and a single-channel analyzer were used to select the $\mathrm{Cu} K \alpha$ wavelength. XRD measurements were also made with a continuous flow cryostat that allowed to investigate the sample at both room and cryogenic temperatures $(8 \mathrm{~K})$. The outer vacuum chamber of the cryostat had aluminized mylar windows for minimum attenuation of x-rays. The cryostat was mounted vertically on the goniometer sample circle, and fine positioning of the sample under vacuum was achieved through a manual linear stage with resolution of $10 \mu \mathrm{m}$. The sample alignment was performed before measurements at both room temperature and $8 \mathrm{~K}$.

\section{RESULTS}

\section{A. Correlation of Tungsten Superconducting Transition Temperature With Deposition Conditions}

Because of the large variability in $\mathrm{W}$ microstructures reported in the literature, the extent to which processing conditions affect the film structure and properties must be experimentally determined in each case. Our initial goal was to reliably produce W thin films with specific $T_{\mathrm{c}}$ values. In order to simplify the problem we have held constant some of the deposition variables such as base pressure $\left(1 \times 10^{-5} \mathrm{~Pa}\right)$, film thickness $(20 \mathrm{~nm})$, substrate bias $(0 \mathrm{~V})$ and temperature $\left(40^{\circ} \mathrm{C}\right)$. We have used a factorial design [11], to study the joint effects of Ar pressure and sputtering power on $\mathrm{W}$ thin films $T_{\mathrm{c}}$ values. The Ar pressure was varied between 0.13 and $1.6 \mathrm{~Pa}$ ( 1 to 12 mTorr), and the deposition power between 150 and $800 \mathrm{~W}$.

The film resistivity and stress were measured immediately after deposition. The films' resistivities varied from 23 to $75 \mu \Omega$. $\mathrm{cm}$. Reported literature values are commonly $20 \mu \Omega \cdot \mathrm{cm}$ or more for $\alpha-\mathrm{W}$ phase and as high as $150-350 \mu \Omega \cdot \mathrm{cm}$ for $\beta$-W phase [6]. Based on these literature values, our as-deposited films have phase compositions varying from pure $\alpha$-W to a mixture of $\alpha-\mathrm{W}$ and a minor amount of $\beta-\mathrm{W}$ phase.

The $T_{\mathrm{c}}$ values were found to correlate with film structure as shown in Fig. 1. The XRD data were in agreement with resistivity values, indicating that the film structure varied from a combination of $\alpha$ and $\beta$ phases for the high $T_{\mathrm{c}}$ film $(500 \mathrm{mK})$

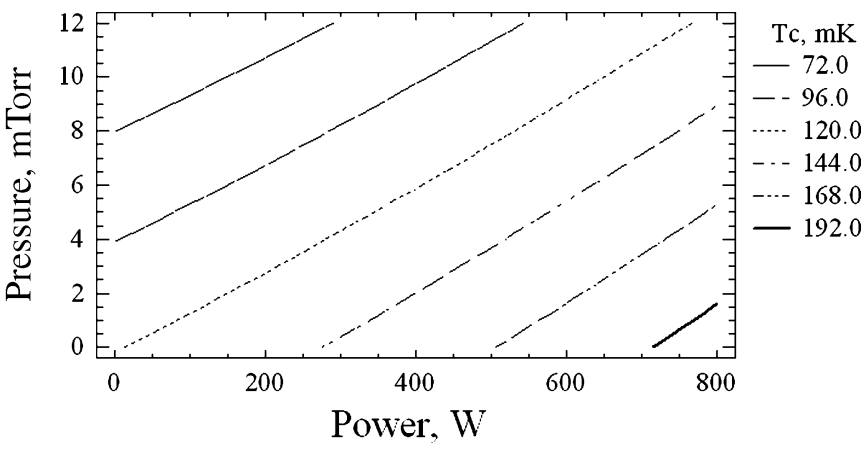

Fig. 2. Contours of estimated response surface for $T_{\mathrm{c}}$ variation with $\mathrm{Ar}$ pressure and dc deposition power.

with high resistivity, to reduced $\beta$-phase component for intermediate values of $T_{\mathrm{c}}$ and resistivities, and finally $\alpha$-phase only for low $T_{\mathrm{c}}$ films (<66 $\mathrm{mK}$ ) with low resistivity. A quantification of the volume fractions was not possible due to the small amount of $\beta$ phase present in the films and the fact that the strongest $\beta(210)$ peak was insufficiently resolved from the $\alpha(110)$ peak, with the resolution of our x-ray system.

Using a statistical software package we determined the dependence of $T_{\mathrm{c}}$ values on the joint effects of Ar pressure and deposition power, Fig. 2.

A multiple linear regression model $\left(\mathrm{R}^{2}=92.36 \%\right)$ can be used to describe $T_{\mathrm{c}}$ dependence on the deposition variables:

$T_{c}=117.21 \mathrm{mK}+0.09 \frac{\mathrm{mK}}{\mathrm{W}} \times P w r-6.08 \frac{\mathrm{mK}}{\mathrm{mTorr}} \times$ Press

Using the fitted model (1), $T_{\mathrm{c}}$ values can be predicted. For our applications we were interested in films with $T_{\mathrm{c}}$ values in the $\sim 100 \mathrm{mK}$ range. The model predicts a $T_{\mathrm{c}}$ value of $121 \pm 12 \mathrm{mK}$ for films deposited at 1.2 $\mathrm{Pa}$ (9 mTorr) Ar pressure and $600 \mathrm{~W}$ deposition power, and the measured value (average of 5 runs) was $109 \pm 10 \mathrm{mK}$. Using this model we were able to tune the $T_{\mathrm{c}}$ of the $\mathrm{W}$ film at values of interest for our applications, through controlled variation of deposition conditions.

\section{B. Underlayer Effect on $W$ Film $T_{\mathrm{c}}$ Values}

$T_{\mathrm{c}}$ measurements in the $100 \mathrm{mK}$ range are inherently timeconsuming and we have attempted to predict the $T_{\mathrm{c}}$ from measurements of other parameters. We have tried to find correlations between the $T_{\mathrm{c}}$ and the film resistivity and stress. However, as previously reported [12], both stress and resistance change over time because of a phase transformation at room temperature.

Using repeated XRD measurements immediately after film deposition, we were able to capture this $\beta$-W to $\alpha$-W phase transformation that stabilized after $\sim 3$ hours (Fig. 3).

The XRD scans also reveal a transition from compressive to tensile film stress state as shown by the shift of (110) $\alpha$-W toward higher $2 \theta$ angles. This stress change has been attributed to the cubical compression $(0.94 \%)$ that results from the $\beta-\mathrm{W}$ to $\alpha$-W phase transformation [8].

The phase transformation is attributed to the metastable nature of $\beta-\mathrm{W}$ phase and also to the energy stored at grain boundaries and at the film-substrate interface [12]. The transformation is thermally driven and can be enhanced by heating. 


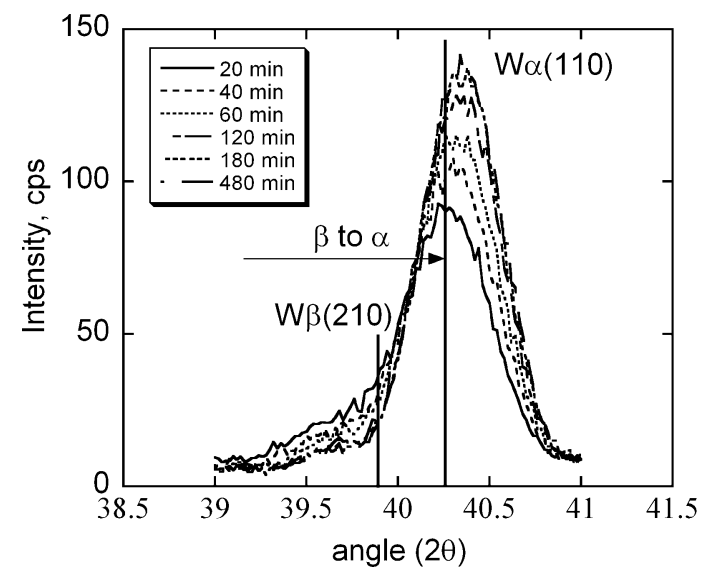

Fig. 3. Room-temperature transformation of $\beta-\mathrm{W}$ phase to $\alpha-\mathrm{W}$ phase in $\mathrm{W}$ film $\left(T_{\mathrm{c}}=110 \mathrm{mK}\right)$.

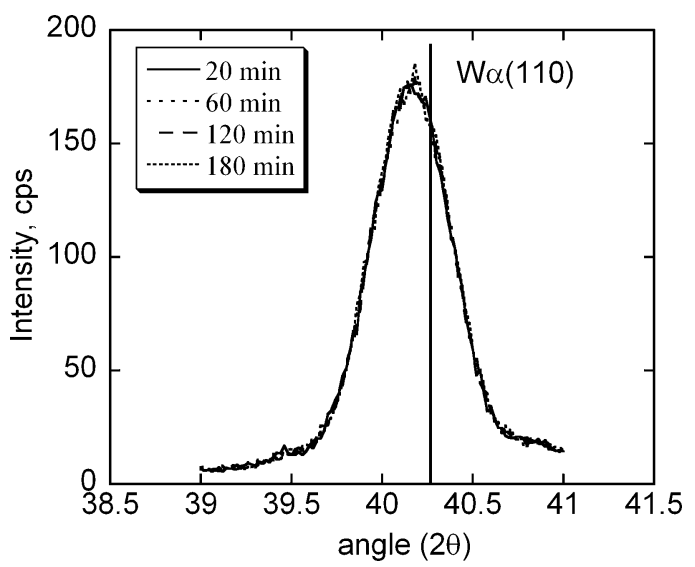

Fig. 4. Stabilization of $\mathrm{W}$ film $\left(T_{\mathrm{c}}=210 \mathrm{mK}\right)$ structure deposited on an in situ amorphous Si layer.

The room-temperature phase transformation is inhibited when the $\mathrm{W}$ film is deposited under identical conditions but on an in situ thin $(10 \mathrm{~nm})$ amorphous Si layer. As shown in Fig. 4 the W film is compressively stressed and stable. The film resistivity is also constant $(23 \mu \Omega \cdot \mathrm{cm})$ as opposed to that of the film deposited directly on a $\mathrm{Si}$ wafer, whose resistivity dropped down to $75 \%$ from deposition until completion of the phase transformation and stabilized at $27 \mu \Omega \cdot \mathrm{cm}$.

The lower resistivity value for the film deposited on in situ amorphous Si corresponds to an increased level of $\alpha-\mathrm{W}$ phase. However, the measured $T_{\mathrm{c}}$ value was $210 \mathrm{mK}$, which is $\sim 100 \mathrm{mK}$ higher than that for the film that underwent the phase transition, and would correspond to increased $\beta$-W fraction. Based on this observation it appears that phase composition is not the only factor determining the $T_{\mathrm{c}}$ value of the film. In this particular case, the only difference between the two films is the presence of the in situ amorphous Si layer for the stable film with higher $T_{\mathrm{c}}$, and a difference in the film stress state as qualitatively revealed by the XRD measurements. Ekin's work [13] on the effect of stress on $T_{\mathrm{c}}$ values for compound superconductors with the $\mathrm{A} 15$ structure, such as $\mathrm{Nb}_{3} \mathrm{Sn}$, indicate that any deviation from zero intrinsic strain in the superconductor results in a reduction of $T_{\mathrm{c}}$. What is intriguing in our case is that the film deposited on amorphous $\mathrm{Si}$, with higher $T_{\mathrm{c}}$, had

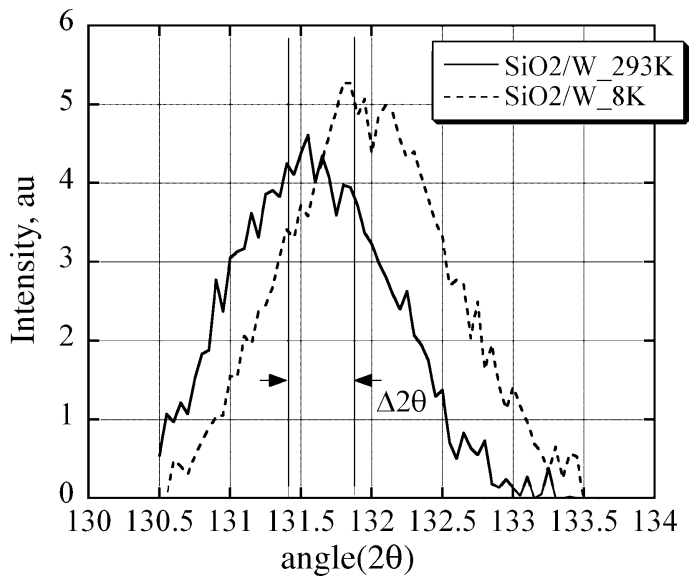

Fig. 5. W (321) peak shift $(\Delta 2 \theta)$ as a result of cooling to $8 \mathrm{~K}$ for $\mathrm{W}$ film on Si substrate with $\mathrm{SiO}_{2}$ coating.

a higher absolute value of compressive stress $(-625 \mathrm{MPa})$, compared to the film on Si wafer, which had a lower tensile stress value ( $\sim 100 \mathrm{MPa}$ ), but also lower $T_{\mathrm{c}}$.

\section{Effect of Antireflective Coating on W Film $T_{\mathrm{c}}$ Values}

We have also observed that when we cover the $\mathrm{W}$ film with an antireflective layer $\left(\mathrm{SiO}_{2}\right.$ or $\left.\mathrm{Si}_{3} \mathrm{~N}_{4}\right)$ the $T_{\mathrm{c}}$ of the film is completely suppressed $\left(T_{\mathrm{c}}<60 \mathrm{mK}\right)$. Again, the film stress state at room temperature was lower for the film whose $T_{\mathrm{c}}$ was suppressed. These observations suggest the possibility of changes in the films as they are cooled to the operating range of our devices. As the films (devices) are cooled from room to cryogenic temperatures, the thermoelastic deformations of the film are constrained by the substrate. Literature data [14] for linear thermal contraction from $300 \mathrm{~K}$ to $4 \mathrm{~K}$ indicate that $\mathrm{W}$ has the highest relative change in length $\left(8.8 \times 10^{-4}\right)$ compared to $\mathrm{Si}$ $\left(2.2 \times 10^{-4}\right)$ and amorphous $\mathrm{SiO}_{2}\left(-0.05 \times 10^{-4}\right)$, which actually displays an anomalous expansion at very low temperatures. These data suggest that when $\mathrm{W}$ film is cooled to cryogenic temperatures, it is constrained by the $\mathrm{Si}$ substrate, which induces a tensile stress in the $\mathrm{W}$ film. When an amorphous $\mathrm{SiO}_{2}$ layer is added on top of a W film on a Si substrate, the W film is now subjected to constraints from both the substrate and the $\mathrm{SiO}_{2}$ layer. We have assumed an added stress effect from the $\mathrm{SiO}_{2}$ coating based on the large difference in $\mathrm{W}(20 \mathrm{~nm})$ and $\mathrm{SiO}_{2}$ $(250 \mathrm{~nm})$ film thicknesses.

Consequently, the differences in thermal contraction by cooling down to cryogenic temperatures may be responsible for a higher stress in the $\mathrm{W}$ film when an $\mathrm{SiO}_{2}$ coating is added, which may explain the suppression of $\mathrm{W}$ film $T_{\mathrm{c}}$. In addition to this hypothesis, structural changes during cooling may also contribute to the $T_{\mathrm{c}}$ variation.

XRD measurements were performed at room temperature and $8 \mathrm{~K}$ for W films deposited on Si substrate with and without a $\mathrm{SiO}_{2}$ coating. We have used the $\mathrm{W}$ peak shifts from room temperature to $8 \mathrm{~K}$ data (Fig. 5) to estimate the induced thermal stress [15]. The $\alpha$-(321) peak was used to estimate the film stress, since high angle peaks are less sensitive to sample displacement errors and more sensitive to strains/stresses.

Based on the peak shifts we were able to calculate the change in $d$-spacing when the films are cooled down to $8 \mathrm{~K}$, using 
Bragg's law, $2 d \sin \theta=\lambda$, where $d$ is the lattice spacing, $\theta$ is the Bragg reflection position, and $\lambda$ is the $\mathrm{Cu} \mathrm{K} \mathrm{K}_{\alpha 1}$ wavelength (1.5406 $\AA$ ). The thermal stress in the film was calculated as the difference between the total stress at $8 \mathrm{~K}$ and room temperature. The film total stress at a particular temperature was calculated as

$$
\sigma(T)=\left(-\frac{1}{2} \frac{E_{\mathrm{f}}}{\nu_{\mathrm{f}}}\right) \times\left(\frac{\Delta d}{d_{0}}\right)_{T},
$$

where $\sigma(T)$ is the film total stress parallel to the film surface, $\mathrm{E}_{\mathrm{f}}$ is the film's Young's modulus, $\nu_{\mathrm{f}}$ is the film Poisson's ratio, and $\left(\Delta d / d_{0}\right)_{T}$, is the film total strain (the change in lattice spacing perpendicular to the film surface, relative to an unstressed tungsten powder) at an arbitrary temperature. Using (2) we found that when cooled from $300 \mathrm{~K}$ to $8 \mathrm{~K}$, the W film on Si substrate is subjected to a thermal tensile stress of $210 \mathrm{MPa}$, whereas the film with the $\mathrm{SiO}_{2}$ coating is subjected to a tensile stress of 1.3 $\mathrm{GPa}$. The above stress values were corrected for sample displacement error [15]. No structural changes were observed by cooling the film to $8 \mathrm{~K}$. This fact was subsequently confirmed by the high-resolution synchrotron data collected at the Advanced Photon Source (APS) at the Argonne National Laboratory.

These results support our assumption that film $T_{\mathrm{c}}$ is affected by thermal stresses when cooled to cryogenic temperatures. In our case we found that the $\mathrm{W}$ film with a suppressed $T_{\mathrm{c}}(<60$ $\mathrm{mK}$ ) is subjected to a thermal tensile stress six times larger as compared to the $\mathrm{W}$ film with $T_{\mathrm{c}} \sim 100 \mathrm{mK}$. Both $\mathrm{W}$ films, with or without the antireflective coating $\left(\mathrm{SiO}_{2}\right)$, were deposited under identical conditions.

\section{CONCLUSIONS}

Using statistical methods we have derived a model able to reliably predict values of $T_{\mathrm{c}}$ for sputter-deposited $\mathrm{W}$ films. The input variables of the model were selected deposition parameters. Apart from deposition conditions, we have demonstrated that the underlayer/coating materials also affect the film $T_{\mathrm{c}}$ values. By deposition of $\mathrm{W}$ on an in situ thin amorphous $\mathrm{Si}$ film, the $\beta$ to $\alpha$ phase transformation was inhibited, leaving the film in a compressively stressed state and increasing the film $T \mathrm{c}$ from $\sim 100 \mathrm{mK}$ to $\sim 210 \mathrm{mK}$. The coating effect on the $T_{\mathrm{c}}$ value was studied through XRD measurements at room temperature and $8 \mathrm{~K}$. The suppression of $T_{\mathrm{c}}$ in coated films is likely to be determined by differential thermal contraction between multilayers, which induced a significant larger tensile stress in the coated films at cryogenic temperatures.

\section{REFERENCES}

[1] B. Cabrera et al., "Detection of single infrared, optical, and ultraviolet photons using superconducting transition edge sensors," Appl. Phys. Lett., vol. 73, p. 735, 1998.

[2] D. Rosenberg et al., Trans. Appl. Supercond., vol. 15, no. 2, Jun. 2005.

[3] A. J. Miller et al., "Demonstration of a low-noise near-infrared photon counter with multiphoton discrimination," Appl. Phys. Lett., vol. 83, pp. 791-793, 2003.

[4] W. L. Bond et al., "Superconductivity in films of $\beta$ tungsten and other transition metals," Phys. Rev. Lett., vol. 15, p. 260, 1965.

[5] S. Basavaiah and S. R. Pollack, "Superconductivity in evaporated tungsten films," Appl. Phys. Lett., vol. 12, p. 259, 1968.

[6] P. Petroff et al., "Microstructure, growth, resistivity, and stresses in thin tungsten films deposited by RF sputtering," J. Appl. Phys., vol. 44, p. 2545, 1973.

[7] I. A. Weerasekera, S. I. Shah, D. V. Baxter, and K. M. Unruh, "Structure and stability of sputter deposited $\beta$-tungsten thin films," Appl. Phys. Lett., vol. 64, p. 3231, 1994.

[8] I. C. Noyan, T. M. Shaw, and C. C. Goldsmith, "Inhomogeneous strain states in sputter deposited tungsten thin films," J. Appl. Phys., vol. 82, p. 4300, 1997.

[9] D. A. Wollman et al., "High-resolution, energy-dispersive microcalorimeter spectrometer for x-ray microanalysis," J. Microsc., vol. 188, p. 196, 1997.

[10] M. E. Huber et al., IEEE Trans. Appl. Supercond., vol. 11, p. 1251, 2001.

[11] D. C. Montgomery, Design and Analysis of Experiments, 5th ed. New York: Wiley, 2001, p. 170.

[12] S. M. Rossnagel, I. C. Noyan, and C. Cabral Jr., "Phase transformation of thin sputter-deposited tungsten films at room temperature," J. Vac Sci. Technol., vol. B 20, p. 2047, 2002.

[13] J. Ekin, "Strain scaling law for flux pinning in practical superconductors. Part 1: basic relationship and application to $\mathrm{Nb}_{3} \mathrm{Sn}$ conductors," Cryogenics, vol. 20, p. 611, 1980.

[14] R. J. Corruccini and J. J. Gniewek, "Thermal Expansion of Technical Solids at Low Temperatures," Nat. Bureau Stand., Monograph 29, 1961

[15] I. C. Noyan and J. B. Cohen, Residual Stress. Measurement by Diffraction and Interpretation. New York: Springer-Verlag, 1987, vol. 120, p. 196. 